\title{
The Simulation Research on Primary District Heating Fluid Network
}

\author{
Nan Cheng ${ }^{1}$, Zhong Liu ${ }^{1}$, Hongmei Zhang1, Lin Xing ${ }^{1}$, Zheng Zhang ${ }^{2, a}$ \\ ${ }^{1}$ State Grid Hebei Electric Power Co., Ltd. Economic Technology Research Institute, China \\ ${ }^{2}$ Hebei University of Engineering, China
}

\begin{abstract}
According to the characteristics of multi-input, multi-output, multi-loop, non-linear and large hysteresis of fluid network, the mathematical model of node, regulating valve and pipeline are established respectively. Simulation based on Panysimu platform is carried out in this paper, and the influence of various disturbances on the entire primary fluid network is analyzed. The simulation results show that the circulation capability of the primary heating fluid network plays a vital role in the rational design of the whole heating system and the safe and economic operation.
\end{abstract}

\section{Introduction}

In view of the current situation of severe haze in northern areas, the development of district heating is of great importance more and more. However, the heat-supply unit is characterized by large initial investment and heating cost. In addition, the heating fluid has multiple input and output, multiple circulation loops, strong coupling and strong hysteresis. In the operation process, there will be various problems, so the dynamic simulation of primary district heating fluid network is very important. The change of the primary side flow affects the resistance coefficient of the primary fluid network, which leads to the re-distribution of the flow and pressure in the primary network. In the case of the increasing heating system, water hammer accidents are likely to occur. Therefore, the analysis of the relationship between the various parameters is the premise of the operation and design of the central heating system.

Hoist J[1and Lawaetz H2et al. conducted in-depth research on dynamic simulation, simulation and control technology of heat source and heat network, and obtained simulation and accurate prediction of dynamic heat load. Hoist Hansson T 3and Lund H T4 et al used program software to simulate the dynamic performance of thermal network. Danish BENONYSSON 5and Zhao6 proposed the "node method" to establish a heat-net model. The method is based on the physical principle and is used to simulate the heat-net flow and dynamic process of each node. Liu tong 7 introduced the modular modeling method into the heating system, and decoupled the large-scale district heating pipe network. A single branch is the basic unit, and each branch unit is divided into pipes and heat exchangers. Based on this assumption the complex heat network can be divided into several modules with different algorithms. Zhang Zheng 8 used $\mathrm{C}++$ Builder to build dynamic simulation software under thermal conditions to analyze the fluid network of the primary heat supply network in the district heating system. He simulated the effect of operating parameters such as node, pipe section flow and circulating pump on the heating network performance, and analyzed the influence on the operation of the heat supply network.

In this paper, the mathematical model of node, regulating valve and pipeline was established according to the operation characteristics of primary heating fluid network. The simulation was carried out based on Panysimu platform, and the influence of various disturbances on the entire primary fluid network was analyzed. The simulation result was of great significance on the reasonable design of thermal network and the safe and economic operation.

\section{The Mathematical model of primary fluid network}

As for district heating system, it generally includes the complex fluid network system which was formed by for the distribution and intersection of the heat medium. Energy transfer and loss process are completed when these heat medium flow through heat transfer stations and pipelines. The dynamic and static characteristics of the fluid network simulation model directly affect the simulation modeling of the whole heating system. Therefore, the fluid network model plays an important role in district heating system model. For the real-time simulation model, the lumped parameter method is usually used to simplify the working fluid network for either single-phase compressible or

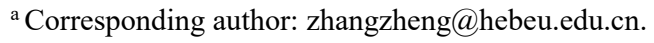


single-phase incompressible fluid.

\subsection{Node model}

In this paper, combined with the thermal dynamic principle and the lumped method, some assumptions can be briefed as follows: the elements of a flow network include branches and nodes. The branch was used to consume energy through a heat exchange station, and the node was used to allocate the flow or energy. However, considering that the flow of fluid in the pipeline was always one-way direction, so it can be simplified as one-dimensional flow. In this case, each node has several branches to influx and outflux. Considering the connection between node and pipe, each pipe in the network has two nodes, and each node has a pipe connection to influx and outflux. In this way, the parameters of each node are determined by the parameters of the adjacent pipe and the corresponding governing equations. The parameters of each pipe were determined by the two nodes of the pipe ends and the corresponding governing equations.

Before establishing the dynamic characteristic equation of node pressure and energy, the following assumptions should be briefed 8 :

In nodes and branches, the flow was considered to be single-phase incompressible, the flow vaporization process was neglected. The flow enthalpy out of the node was equal to the flow enthalpy at the node.

There is no mass transfer between branch and nodes and the external environment, but energy transfer with the external environment. That is, there is heat loss between nodes and pipelines.

The pressure dynamic equation and energy dynamic equation of the nodes are deduced by taking the most representative $\mathrm{N}$-branch branches flowing into the $\mathrm{N}$-branch flows out as an example.

Derivation of dynamic equation of nodal pressure

The node pressure model is illustrated as follows:

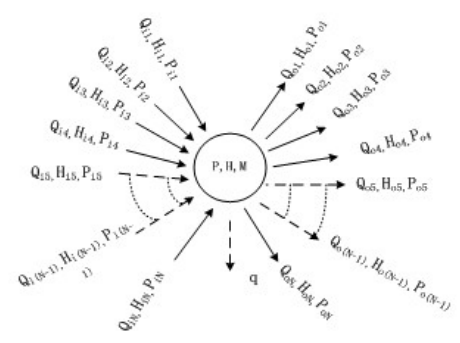

Figure1. Diagram of node

Mass conservation equation of node:

$\frac{d(\rho V)}{d t}=\sum_{n=1}^{n=N} Q_{i n}-\sum_{n=1}^{n=N} Q_{o n}$

Expand the left side of the above formula

$\rho \frac{d V}{d t}+V \frac{d \rho}{d t}=\sum_{n=1}^{n=N} Q_{i n}-\sum_{n=1}^{n=N} Q_{o n}$
The capacity of the node can be assumed to be constant,

that is, the flow volume keep constant, then there is $\frac{d V}{d t}=0$, from the Formula (2)

$V \frac{d \rho}{d t}=\sum_{n=1}^{n=N} Q_{i n}-\sum_{n=1}^{n=N} Q_{o n}$

Pressure and enthalpy are independent variables of density, namely, $\rho=f(p, H)$. There are

$V \frac{d \rho}{d t}=V\left(\frac{\partial \rho}{\partial P} \cdot \frac{\partial P}{\partial t}+\frac{\partial \rho}{\partial H} \cdot \frac{\partial H}{\partial t}\right)=\sum_{n=1}^{n=N} Q_{i n}-\sum_{n=1}^{n=N} Q_{o n}$

And suppose, $\frac{\partial \rho}{\partial H} \cdot \frac{\partial H}{\partial t} \approx 0$, there are

$V \frac{d \rho}{d t}=V\left(\frac{\partial \rho}{\partial P} \cdot \frac{\partial P}{\partial t}\right)=\sum_{n=1}^{n=N} Q_{i n}-\sum_{n=1}^{n=N} Q_{o n}$

Define $V \cdot \frac{\partial \rho}{\partial P}=K$ as a nonzero compressible coefficient; the Equation (5) is turned into:

$K \cdot \frac{\partial P}{\partial t}=\sum_{n=1}^{n=N} Q_{i n}-\sum_{n=1}^{n=N} Q_{o n}$

The flow formula $W=F \cdot \sqrt{\Delta P}$, derived from the Bernoulli equation, is added to (6)

$K \cdot \frac{d P}{d t}=\sum_{n=1}^{n=N} F_{\text {in }} \cdot \sqrt{P_{n 1}-P}-\sum_{n=1}^{n=N} F_{\text {on }} \cdot \sqrt{P-P_{\text {on }}}$

The implicit Euler method is applied to Equation (7)

$K \frac{P(k+1)-P(k)}{d t}=\sum_{n=1}^{n=N} F_{\text {in }} \cdot \sqrt{P_{\text {in }}(k+1)-P(k+1)}-\sum_{n=1}^{n=N} F_{\text {on }} \cdot \sqrt{P(k+1)-P_{\text {on }}{ }^{(k+1)}}(8)$

$\mathrm{P}(\mathrm{k}+1)$ cannot be derived directly from (8), and the nonlinear part needs to be linearization

$F_{i 1} \cdot \sqrt{P_{i 1}(k+1)-P(k+1)}=F_{i 1} \cdot \frac{P_{i 1}(k+1)-P(k+1)}{\sqrt{P_{i 1}(k+1)-P(k+1)}}$

In order to give a linear processing definition to branch

$R_{i 1}(k)=F_{i 1} \cdot \frac{1}{\sqrt{P_{i 1}(k)-P(k)}}$

Finally get

$$
P(k+1)=P(k)+\frac{\sum_{n=1}^{n=N} R_{i n}\left[P_{i n}(k)-P(k)\right]-\sum_{n=1}^{n=N} R_{\text {on }}\left[P(k)-P_{\text {on }}(k)\right]}{\frac{K}{d t}+\sum_{n=1}^{n=N} R_{\text {in }}+\sum_{n=1}^{n=N} R_{\text {on }}}
$$

Derivation of dynamic equation of nodal energy

As shown in Figure1, considering that there is a certain volume $\mathrm{V}$ and a certain mass $\mathrm{m}$ at the node, the energy conservation equation at the node is as follows:

$$
\frac{d M H}{d t}=\sum_{n=1}^{n=N} Q_{i n} H_{i n}-\sum_{n=1}^{n=N} Q_{o n} H-q
$$


$M \frac{d H}{d t}+H \frac{d M}{d t}=\sum_{n=1}^{n=N} Q_{i n} H_{i n}-\sum_{n=1}^{n=N} Q_{o n} H-q$

Obtained by the mass conservation equation

$$
\frac{d M}{d t}=\sum_{n=1}^{n=N} Q_{i n}-\sum_{n=1}^{n=N} Q_{o n}-q
$$

Substitute (13) into (12) and get

$M \frac{d H}{d t}=\sum_{n=1}^{n=N} Q_{i n}\left(H_{i n}-H\right)-q$

When the first-order implicit Euler method is used, the variation of the Formula (15) is followed as

$$
M \frac{H(k+1)-H(k)}{d t}=\sum_{n=1}^{n=N} Q_{i n}\left[H_{i n}-H(k+1)\right]-q
$$

Collate, and get the final formula

$$
H(k+1)=H(k)+\frac{\sum_{n=1}^{n=N} Q_{i n}\left[H_{i n}-H(k)\right]-q}{\frac{M}{d t}+\sum_{n=1}^{n=N} Q_{i n}}
$$

\subsection{Regulating valve model}

The electric regulating valve is used in the system model established in this paper. The flow of the regulating valve was regulated by changing the local resistance. When the mathematical model of regulating valve is established, the flow capacity and flow characteristics of valve are analyzed. The main parameters of the electric regulating valve are described below:

Valve flow capacity

When the differential pressure between the two ends of the valve is $0.1 M P a$, the flow rate through the valve per unit time namely is the flow capacity of the valve, in terms of the symbol $K v$; when the fluid capacity is at maximum, it should be expressed as a symbol $K v s$. As shown in the following formula:

$$
K v=\frac{316 Q}{\sqrt{\frac{p_{1}-p_{2}}{\rho}}}, K v=\frac{316 G}{\sqrt{p_{1}-p_{2} \rho}}
$$

Where $Q$ :volumetric flow rate, $m^{3} / h ; G$ :mass flow, ${ }^{t / h} ;{ }^{p_{1}},{ }^{p_{2}}$ :Absolute pressure before valve, Absolute pressure behind the valve, $\mathrm{Pa}$; ${ }^{\rho}$ :The liquid density, $\mathrm{g} / \mathrm{cm}^{3}$.

Flow characteristics of valve

The relationship between the relative flow rate of the valve and the relative opening of the valve is the flow characteristic of the valve. The mathematical expression is:

$$
\frac{Q}{Q_{\max }}=f\left(\frac{L}{L_{\max }}\right)
$$

In the formula: $Q$ is the valve flow rate at any open degree; $Q_{\max }$ is the flow rate at full opening of valves; $L$ is open valve stroke corresponding to the $Q ; L_{\max }$ is full opening valve stroke.

Pressure drop on both sides of the valve is variable during field operation, the valve flow characteristic obtained when pressure drop changes on both sides of the valve is called working flow characteristic. Considering the pressure difference on the valve, Equation (19) can be changed to:

$$
\frac{Q}{Q_{\max }}=f\left(\frac{L}{L_{\max }}\right) \cdot \sqrt{\frac{\Delta p}{\Delta p \max }}
$$

Among the formula, $\Delta p$ is the differential pressure between the two sides of the regulating valve for a certain opening, $M P a ;{ }^{\Delta p} \max$ is the differential pressure between the two sides of the regulating valve for full opening, $\mathrm{MPa}$;

According to Equation (18):

$\frac{Q}{Q_{\max }}=\frac{K v}{K v s} \cdot \sqrt{\frac{\Delta p}{\Delta p}} \cdot \sqrt{\frac{\rho_{\max }}{\rho}}$

Ignoring changes in the density of water, it can assume $\rho_{\max }=\rho$, then

$\frac{Q}{Q_{\max }}=\frac{K v}{K v s} \cdot \sqrt{\frac{\Delta p}{\Delta p_{\max }}}$

Combine the Formula (20) and (22), get

$\frac{K v}{K v s}=f\left(\frac{L}{L_{\max }}\right) \Rightarrow K v=K v s \cdot f\left(\frac{L}{L_{\max }}\right)$

Therefore, through the Kvs value and the ideal flow characteristic curve of the regulating valve, it can figure out the value of $K v$ at some opening. Then from formula $Q=K v \cdot \sqrt{\Delta p}$, the outlet flow of the control valve is

$$
Q=K v s \cdot f\left(\frac{L}{L_{\max }}\right) \cdot \sqrt{\Delta p}
$$

\subsection{Pipeline model}

The heat-supply pipeline can be divided into two sections. As shown in Figure2.

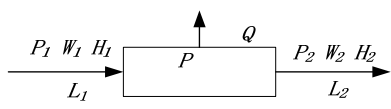

Figure2. Model of a pipeline 
It is commonly believed that the working medium in the container is motionless, and the temperature is varying. The temperature is determined by the temperature of the vessel. Flow loss and flow rate can be calculated by the pipeline governing equations.

As shown in Figure3, the forces acting on the fluid differential unit are flow pressure difference, gravity and friction resistance, and their projections in the flow direction are respectively $-\frac{\partial p}{\partial x} \Delta x A$, $-\rho g A \Delta x \sin \alpha,-\frac{\lambda}{D} \Delta x \frac{1}{2} \rho V^{2} A$.

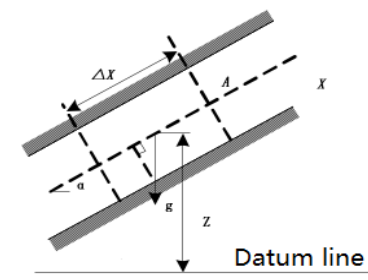

Figure3. Isolated body of motion equation

The acceleration in the flow direction denoted by Euler method is

$\left[\frac{d V}{d t}\right]_{x}=\frac{\partial V}{\partial t}+V \frac{\partial V}{\partial x}$

According to Newton's second law

$$
\frac{\partial V}{\partial t}+V \frac{\partial V}{\partial x}+g \sin \alpha+\frac{1}{\rho} \frac{\partial P}{\partial x}+\lambda \frac{V^{2}}{2 D}=0
$$

According to the conservation of mass

$$
\frac{d(\rho A \Delta x)}{d t}=0
$$

Namely

$\frac{1}{\rho} \frac{\partial \rho}{\partial t}+\frac{1}{A} \frac{d A}{d t}+\frac{1}{\Delta x} \frac{d(\Delta x)}{d t}=0$

By using the modulus of elasticity of volume $K=\frac{\Delta P}{\frac{\Delta \rho}{\rho}}$

The relative rate of change of the length of the fluid differential unit can be expressed as

$$
\frac{1}{\Delta x} \frac{d(\Delta x)}{d t}=\frac{\partial V}{\partial x}
$$

The relative change rate of the cross section of the micro fluid is related to the elasticity of the pipe wall and the constraint conditions of the pipe:

$$
\frac{1}{A} \frac{d A}{d t}=\frac{P D}{E e} C_{1}
$$

Where $\mathrm{E}$ represents the elastic modulus of pipe, e represents the thickness of pipe wall, and $\mathrm{C} 1$ is the coefficient related to the constraint conditions of the pipe. Substitute equations (29), (30) and (31) into equations (28)
$\frac{1}{K} \frac{d p}{d t}+\frac{d p}{d t} \frac{D C_{1}}{E e}+\frac{\partial V}{\partial x}=0$

Make

$$
a^{2}=\frac{\frac{K}{\rho}}{1+\frac{K D}{E e} C_{1}}
$$

Then

$$
\frac{1}{\rho} \frac{d p}{d t}+a^{2} \frac{\partial V}{\partial x}=0
$$

\section{Simulation results and analysis}

\subsection{Effect of the steam inlet valve opening on primary fluid network}

When the system runs stably, if the opening of the steam valve of $\# 1$ and $\# 2$ heaters decreases from $100 \%$ to $80 \%$, the steam extraction parameters and heater parameters change. Within a short period of time, the total amount of steam extraction quantity from heating has a linear downward trend. A slight increase come after a sharp decrease to a certain value, and then the steam quantity at last reaches the equilibrium state after rising to a certain value with the pressure no longer changing. The temperature of steam extraction begins to soar up at the moment when the steam quantity drops sharply. When the quantity of steam exhaust reaches equilibrium, it also gradually reaches the equilibrium state. The extraction steam enthalpy increased slightly and reached steady state in a short time. As shown in Figure4.

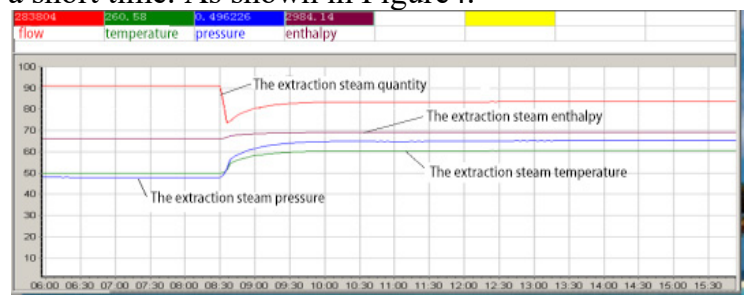

Figure4. Variation diagram of extraction parameters

This is because when adjust the valve opening degree from $100 \%$ to $80 \%$, the total amount of steam entering the heater \# 1 and \# 2 reduced significantly. And this wills feedback an excessive signal. Then the total amount of extraction steam decreases rapidly, the quantity of supply comes even lower than that of the demand. After a short period buffering, the steam amount begin to increase. And gradually keep constant. The steam amount flowing into heater \#1 and heater \#2 decreases, and the steam that should flow into heaters now accumulates in the pipe, which will immediately result in the inlet pressure increasing. In order to keep the temperature of water supply in the heat network, the temperature of steam extraction has to be increased responding to the reduction of steam extraction. This will inevitably lead to the increase of 
extraction enthalpy for the close relationship of steam enthalpy and the temperature.

And when the opening of the inlet steam valve decreases, the pressure of $\# 1$ heater and $\# 2$ heater decreases, the outlet temperature decreases, the pressure of \#3 heater and \#4 heater increases, and the outlet temperature increases. As shown in Figure5 and Figure 6.

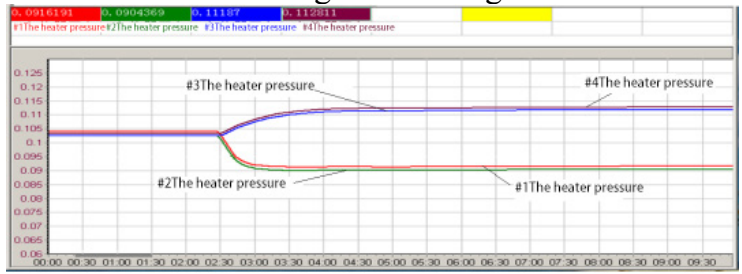

Figure5. Diagram of pressure change of heater

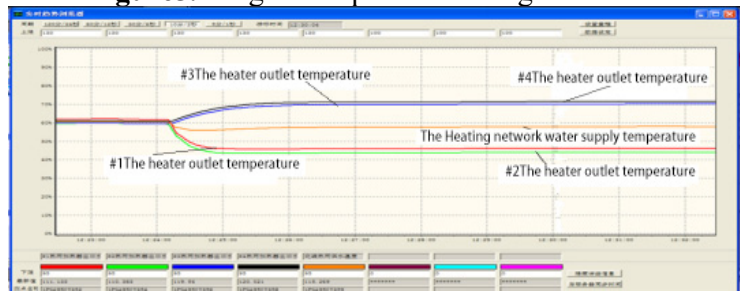

Figure6. Shows the temperature change at the outlet of the heater

For the amount of steam in \#1 and \#2 heaters decreases, in order to maintain the temperature of water supply, the amount of steam in \#3 and \#4 heaters increases. According to the gas state equation $\mathrm{PV}=\mathrm{RT}$, the pressure of $\# 1$ and \#2 heaters decreases and the pressure of \#3 and \#4 heaters increases. At the same time, with the same amount of steam flowing into the heater, the heat transfer decreases and the outlet temperature decreases. Similarly, the reasons for the rising temperature at the outlet of \#3, \#4 heaters can be explained. If the total return water is constant and the total supply of steam decreases, the total water supply temperature will decrease accordingly.

\subsection{Effect of flow Inlet Valve Opening on Primary Fluid Network}

When the system operates stably, the opening of the \#1 circulating water pump's water valve changes from $100 \%$ to $80 \%$, the circulation pump outlet flow, the heater outlet temperature, the Heating network water supply temperature and flow are all changed. The circulation pump outlet flow of \#1 decreases sharply, while the circulation pump outlet flow of \#2 and \#3 increases sharply, and the total water supply decreases, as shown in Figure7.

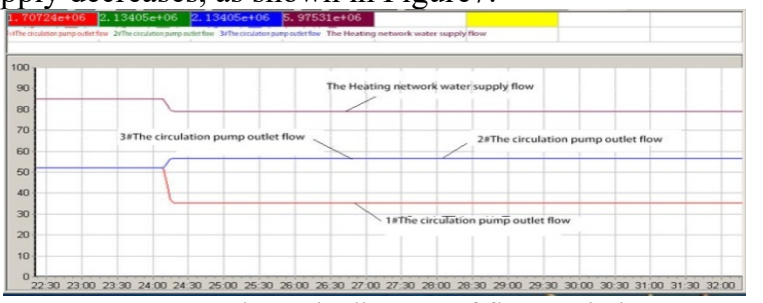

Figure7. Schematic diagram of flow variation

This is due to the decrease in the valve opening, which reduces the bypass flow capacity and the flow to the \#1 circulating pump is also decreased. In order to keep the water supply in stable condition, the flow of circulating pump \# 2 and \# 3 should increase. However, the flow capacity of the valve is limited by the rating capacity of the valve. The amount of water into the $\# 2, \# 3$ circulating pump is not enough to make up for the decreasing amount of \#1 circulating pump, and in this condition, the total water supply decreases.

When the opening of the water valve of \#1 circulating pump changes $\# 1, \# 2, \# 3$, \#4 heater outlet temperature and heating network water supply temperature increase slowly to a certain value, it tends to be stable as shown in Figure8.

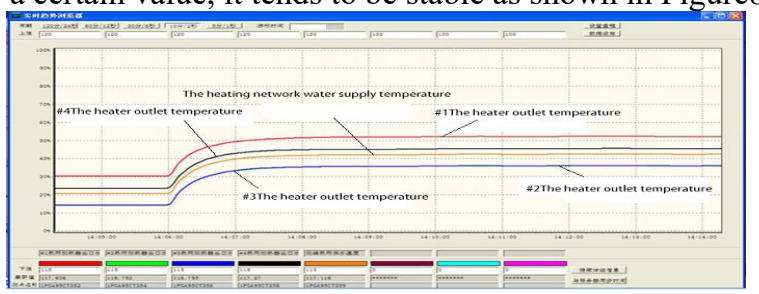

Figure8. Diagram of temperature variation

This is because the flow reduction into \#1 circulation pump leads to a reduction into the main pipe of the circulating pump and a reduction in the flow of water allocated to the four heaters. When the steam supply is constant, the heat transfer increases, the heater outlet temperature increases, and the heating network water supply temperature also increases accordingly.

\subsection{Effect of steam trap e opening on Primary Fluid Network}

When the system is in stable condition if the opening of the steam trap valve increase from $50 \%$ to $60 \%$, the heater parameters and water supply temperature change, the water level in heater drops rapidly, and as time goes on, the water level drops to zero, as shown in Figure9.There is a slight increase in the heater outlet temperature and the heating network supply temperature, as shown in Figure10.

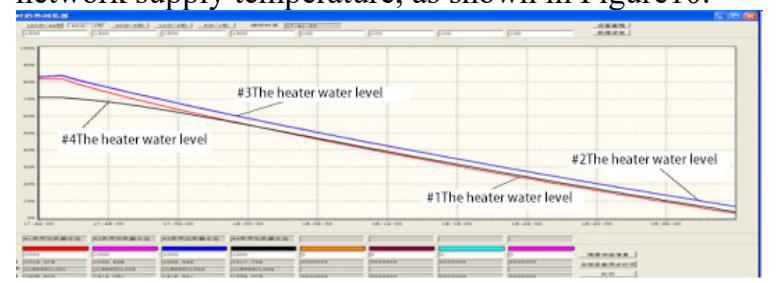

Figure9. Diagram of water level change of heater

The reason for the drop of water level in the heater is that the steam trap valve increases suddenly and the water in the heater pour down rapidly. While the steam entering the heater has not yet condensed into water, which will causes the heater water level to drop continuously. 


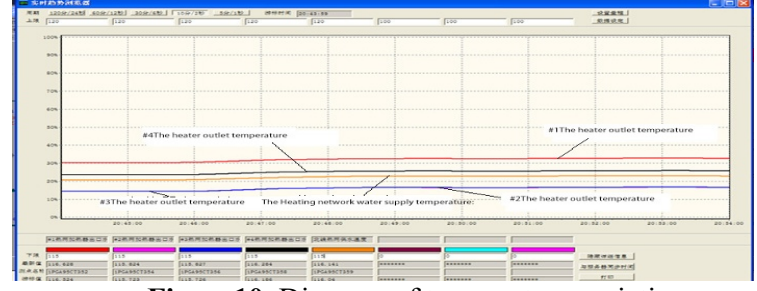

Figure10. Diagram of temperature variation

The temperature rise is due to the increase of the drain capacity, the water reduction on the shell side and the enhancement of the heat transfer between the steam on the shell side and the water in the pipe. At the same time, the rapid discharge of drain reduces the amount of heat taken away. Therefore, the temperature in the heater will increase slightly, and the temperature of the total heat grid will also increase.

\section{Conclusions}

The mathematical model of primary district heating system network was established in this paper, then the system performance with different conditions was studied through the Panysimu simulation platform. The research shows that when the flow capacity of one branch changes, it will affect the flow of the other branches, especially the adjacent branches, which is easy to lead to the hydraulic and thermal maldjustment. Therefore, the analysis of the influence of primary fluid network can prevent the phenomenon of branch hydraulic misadjustment effectively, which has a good reference function for the safe and economical operation of heating system.

\section{Reference}

1. Hoist J . Madsen H, Hansen F.M. Estimation of continuous time models for the heat dynamics of a building. Denmark: Lurid institute of Technology, 1990.

2. Lawaetz H.B.Bohm B.System for production, distribution and heat demand Denmark: Technological institute of Copenhagen, 1984.

3. Hansson T.R Operational optimization of district heating systems. Denmark: Technological University of Denmark, 1991.

4. Lund H.T. Methods for operational optimization of district heating systems . Denmark: The allergy research cooperation of the Nordic Council of Ministers, 1989.

5. A.Benonysson. Dynamic Modeling and Operational Optimization of District Heating Systems. PhD Thesis at Laboratory of Heating and Air Conditioning, Technical University of Denmark.1991:42-87

6. H.Zhao.Analysis. Modeling and Operational Optimization of District Heating Systems. PHD Thesis at Laboratory of Heating and Air Conditioning, Technical University of Denmark. 1995: 30-41

7. Liu Tong. Advanced control simulation and realization of large central heating pipe network [D]. Master's thesis of Beijing institute of architecture and engineering.2008:16-18

8. Zhang Zheng. Simulation of the Dynamic Performance and Research on the Control and Optimization for the District Heating System [D]. Master's thesis of Shandong University.2011 